University of Massachusetts Amherst

ScholarWorks@UMass Amherst

\title{
The Mechanics of the Bicycle Pedal Photoisomerization in Crystalline cis,cis-1,4-Diphenyl-1,3-butadiene
}

\author{
Cody R. Aldaz \\ University Of Michigan \\ Todd J. Martínez \\ Stanford University \\ Paul M. Zimmerman \\ University of Michigan
}

Follow this and additional works at: https://scholarworks.umass.edu/muri_pubs

Aldaz, Cody R.; Martínez, Todd J.; and Zimmerman, Paul M., "The Mechanics of the Bicycle Pedal Photoisomerization in Crystalline cis,cis-1,4-Diphenyl-1,3-butadiene" (2020). The Journal of Physical Chemistry A. 25.

https://doi.org/10.1021/acs.jpca.0c05803

This Article is brought to you for free and open access by the MURI on Photomechanical Materials at ScholarWorks@UMass Amherst. It has been accepted for inclusion in Publications by an authorized administrator of ScholarWorks@UMass Amherst. For more information, please contact scholarworks@library.umass.edu. 


\section{The Mechanics of the Bicycle Pedal Photoisomerization in Crystalline}

\section{Abstract} conversion.

Cody R. Aldaz, Todd J. Martínez, and Paul M. Zimmerman

Direct irradiation of crystalline cis, cis-1,4-diphenyl-1,3-butadiene (cc-DPB) forms trans, trans1,4-diphenyl-1,3,-butadiene via a concerted two bond isomerization called the bicycle pedal mechanism. However, little is known about photoisomerization pathways in the solid-state and there has been much debate surrounding the interpretation of volume-conserving isomerization mechanisms. The bicycle pedal photoisomerization is investigated using the QM/MM complete active space self-consistent field (CASSCF)/Amber force-field method. Important details about how the steric environment influences isomerization mechanisms are revealed including how the one-bond flip and hula-twist mechanisms are suppressed by the crystal cavity, the nature of the seam space in steric environments, and the features of the bicycle pedal mechanism. Specifically, in the bicycle pedal the phenyl rings of cc-DPB are locked in place and the intermolecular packing allows passageway for rotation of the central diene in a volume-conserving manner. In contrast, the bicycle pedal rotation in the gas-phase is not a stable pathway, so single-bond rotation mechanisms become operative instead. The present models, however, do not capture the quantitative activation barriers and more work is needed to better model reactions in crystals. Lastly, the reaction barriers of the different crystalline conformations within the unit cell of cc-DPB are compared to investigate the possibility for conformation-dependent isomerization. Although some difference in reaction barriers are observed, the difference is most likely not responsible for the experimentally observed periods of fast and slow

\section{Introduction}

The ability of light to trigger mechanical changes via photoisomerization is well known in vision and phototaxy, driving efforts to replicate these features in artificial molecular machines and 
1 optomechanical devices. This has led to the

2 study of specific isomerization mechanisms

3 such as one-bond flip (OBF, also known as

4 the double-bond twist), volume-conserving

5 hula-twist (HT), and the bicycle pedal (BP)

6 mechanisms $^{1}$. The relative importance of

7 these photoisomerization steps in

8 condensed phases, however, is particularly

a)

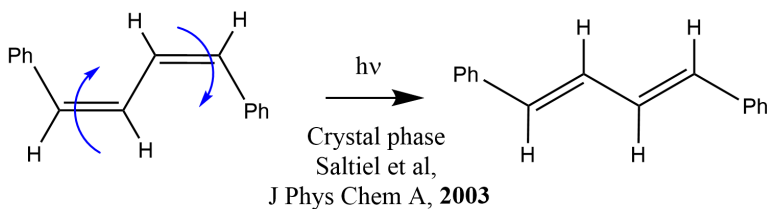

b)

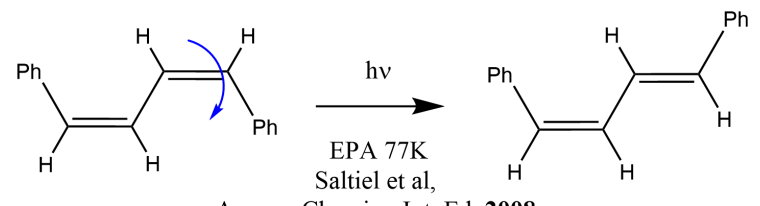

Angew. Chemie - Int. Ed, 2008

Figure 1 Irradiation of cc-DPB in different steric environments results in different isomerization products a) crystal b) viscous environment.

9 sensitive to the steric environment.

10 Furthermore, the study of these mechanisms is notoriously difficult because thermal isomerization

11 about carbon-carbon single bonds can mix the photoproducts. As a result, many questions and

12 debate $^{2-6}$ surround the interpretation of photoisomerization mechanisms, and this confusion inhibits

13 rational design.

Herein, the isomerization of crystalline cis,cis-1,4-diphenyl-1,3-butadiene (cc-DPB), ${ }^{7,8}$ to trans,trans-1,4-diphenyl-1,3-butadiene (tt-DPB) is investigated (Figure 1a). This isomerization may

16 proceed through the concerted BP mechanism, which has been noted in crystalline butadienes ${ }^{9-11,12}$

17 (some of which produce macroscopic motion like peeling ${ }^{12}$ ), hexatrienes ${ }^{13,14}$, photoactive yellow

18 protein $^{15}$, and has been implicated in the visual retinoid cycle ${ }^{16}$. Photoirradiation of crystalline cc-DPB

19 is interesting among these because in solution and glassy phase the BP isomerization is minor or

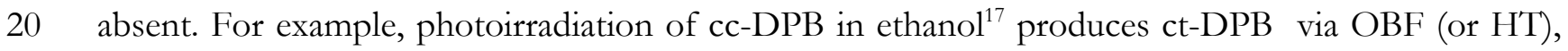

21 and a minor amount of tt-DPB through a phenylallylbenzyl intermediate, not through concerted

22 rotation as portrayed in the BP mechanism ${ }^{17}$. Furthermore, cc-DPB in viscous media like glassy phases

23 mostly does not undergo BP isomerization: photoirradiation in the viscous isopentane glass resulted

24 in a mixture of single double-bond isomerization and BP isomerization ${ }^{7}$, whereas photoirradiation in 25 the harder EPA (ether:isopentane:ethyl alcohol=5:5:2) glass resulted in no BP isomerization(Figure

$26(\mathrm{~b})^{18}$. This is unexpected because isomerization in volume-restricting environments is generally

27 expected to proceed via the more volume-conserving mechanism (e.g. HT/BP vs OBF) ${ }^{1}$. These

28 observations therefore suggest that specific steric and structural interactions must be present in the

29 crystal which inhibit the single double-bond isomerization and enable the BP pathway.

In order to gain more information into this intriguing mechanism, it is useful to perform $a b$

31 initio computational analysis. Previous studies on related compounds ${ }^{19-25}$ have demonstrated that 
1 quantum chemical simulations can provide important details of photoisomerization mechanisms that

2 would be useful for understanding cc-DPB photochemistry. Specifically, conical intersections

3 (crossings between potential energy surfaces) enable efficient non-radiative decay between states, and

4 therefore control photoproduct formation. Quantum mechanical studies of conical intersections

5 responsible for the BP mechanism, however, are to the best of our knowledge unexplored.

6 Furthermore, the investigation of reaction mechanisms in crystal environments is more difficult than

7 their gas-phase counterparts. Zimmerman was the first to report ab initio investigations of crystal

8 photochemical reactions by approximating crystal packing with an inert gas-shell model ${ }^{26}$. Later, the

9 same author optimized cluster models using a quantum mechanics/molecular mechanics (QM/MM)

10 ONIOM approach ${ }^{27,28}$. Recent advances in related techniques applied to crystal photochemistry ${ }^{29,30}$

11 have permitted improved geometry optimization as well as better treatment of short-range ${ }^{31}$ and long-

12 range electrostatic effects ${ }^{32,33}$ in crystals with quantum mechanics/quantum mechanics' methods

13 (QM/QM') methods.

14 In this article, a recently developed reaction path optimization method ${ }^{34,35}$ is applied to analyze 15 photoisomerization of cc-DPB. First, analysis of traditional OBF double-bond isomerization 16 pathways provides insight into why these pathways are forbidden in crystals. Details of the BP 17 mechanism for DPB in crystal are then revealed for the first time. The descriptions of these pathways 18 will reveal how the crystal phase affects conical intersections and on how the broader seam topography 19 depends on restrictions on intramolecular rotation. Finally, a hypothesis about the isomerization rate 20 varying on a conformer-specific level is evaluated ${ }^{8,36,37}$. Altogether, this information provides a close 21 look at the effects of environment on photoisomerization mechanisms and explains how qualitative 22 changes in reaction pathways can come with entry of molecules into the crystal phase.

Methods:

\section{Cluster model}

The cc-DPB crystal structure was retrieved from the Cambridge crystal database (CIF \#29021) ${ }^{7}$. A 3x3x3 cluster was prepared and a four-layer QM/MM approach was utilized for all 28 calculations unless otherwise stated (Figure 2). Ordinarily, there are three layers in the crystal cluster models: QM, MM, and MM frozen ${ }^{27,28}$. An extra layer arises here because of the hybrid Cartesian and translation-rotation internal coordinate (TRIC) system which enables efficient coordinate 
1 optimization and transformation (see

2 below $)^{35}$. The choice of layers is as follows.

3 The first layer contains the QM molecule,

4 which depends on the specific

5 conformation of the unit cell being

6 investigated (see below). The second layer

7 is defined as all monomers with all atoms

8 less than $5 \AA$ from the QM monomer and

9 is treated with MM and TRIC. The third

10 layer is defined as monomers with all

11 atoms greater than $5 \AA$ away from the QM

12 monomer and less than $10 \AA$ from the QM

13 monomer and is treated with MM and

14 Cartesians. The fourth layer is all

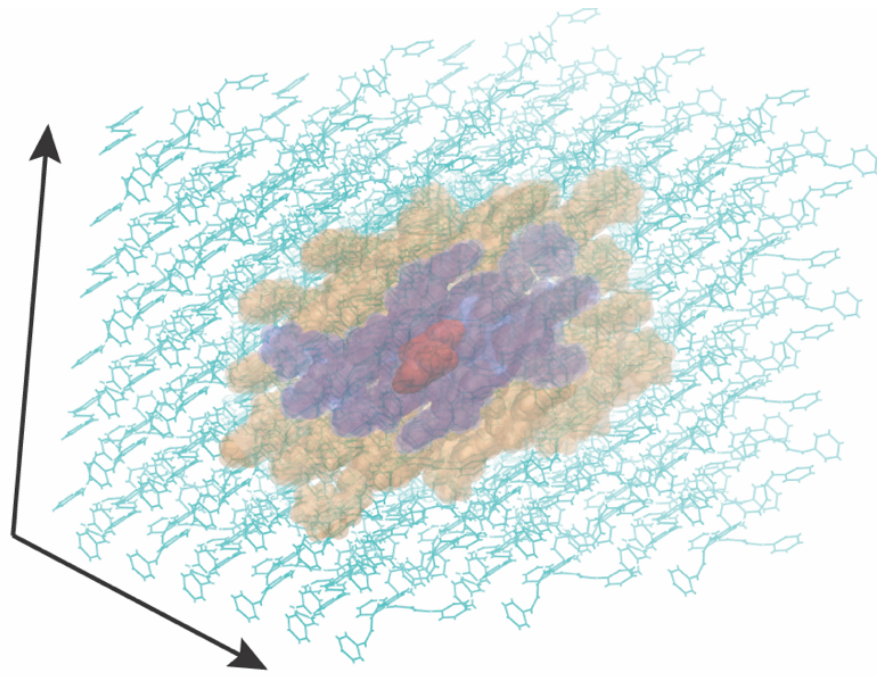

Figure 2. $3 \times 3 \times 3$ cluster model of cc-DPB. The colors represent the fourlayer geometry optimization approach. Red VDW: non-frozen QM region treated with TRIC, blue VDW: non-frozen MM region treated with TRIC, transparent VDW orange: non-frozen MM region treated with Cartesians, line cyan: frozen MM region treated with Cartesians. There are 12960 atoms total.

15 monomers with all atoms greater than $10 \AA$ from the QM monomer; this layer is kept frozen and is treated with Cartesian coordinates. The QM layer is treated with the state-averaged complete active

17 space self-consistent field (SA-CASSCF) method. All layers besides the QM layer are treated with the

18 Amber14 force field and utilize restrained electrostatic potential charges (RESP) derived from 19 quantum mechanics. The atom types and charges are provided in the Supporting Information. The 20 choice of active space in the SA-CASSCF is discussed below because that requires further 21 experimental and theoretical considerations.

22 The use of frozen atom boundary conditions was investigated by comparing the BP pathway 23 (cc-DPB $\rightarrow$ tt-DPB) in the ground-state with and without frozen boundary conditions using the MM 24 level of theory (Supporting Information). No significant difference in the ground-state BP pathway 25 was observed for the frozen-atom vs no frozen atom simulation. Therefore, because the present 26 implementation of QM/MM must rely on frozen boundary conditions or some other type of restraints 27 to maintain crystal packing, freezing the outer layer of the crystal is not expected to affect the excited28 state QM/MM simulations.

Reaction path optimization was performed with the growing string method (GSM) as 31 implemented in Python $3^{38}$. The core GSM algorithm has been described separately ${ }^{39,40}$, and 
modifications necessary to treat large systems will be described in detail elsewhere ${ }^{34}$ Therefore, only

2 a brief description is given here. GSM is an efficient reaction path optimization algorithm that can be operated in either single-ended or double-ended mode. Single-ended mode requires an initial structure and a driving coordinate direction (e.g. adding or breaking bond) and is therefore well suited for reaction discovery and exploration ${ }^{41}$. This mode has also been shown to be useful for finding MECI starting from stable equilibrium geometries ${ }^{42}$. The double-ended mode requires an initial and final structure as input. GSM employs delocalized internal coordinates (DLCs) which are superior to Cartesian coordinates for geometry optimization ${ }^{43}$. In the present implementation of GSM, translation and rotation internal coordinates (TRIC) ${ }^{35}$ are used to reduce the computational cost of coordinate transformations. With the inclusion of TRIC, the individual molecules of the cluster are decoupled and therefore block-matrix diagonalization techniques are used to achieve linear scaling (in the number of molecules, $N_{m o l}$ ) computational $\operatorname{cost}^{34}$. Further reductions in cost for coordinate transformation are obtained by treating a subset of the atoms with Cartesian coordinates (see

\section{Supporting Information).}

Python GSM is available for free on Github ${ }^{38}$ and is interfaced with GPU-accelerated TeraChem for QM and QM/MM calculations ${ }^{44-46}$, and OpenMM for MM calculations ${ }^{47}$. Initial geometry optimization of the $3 \times 3 \times 3$ crystal was performed with OpenMM without the frozen constraints to relax the MM region. MECI optimization was performed using an iterative penalty potential $^{48}$. Gradient RMS, gradient maximum, and energy difference criteria were used to determine optimization convergence. Transition state optimization was performed using a climbing image algorithm $^{39}$. The transition states are considered optimized when the gradient RMS and reaction tangent are converged to $0.0005 \mathrm{Ha} /(\mathrm{Bohr} \mathrm{rad})$, and the difference in energy between successive iterations was less than $0.1 \mathrm{kcal} / \mathrm{mol}$. Seam saddle point were optimized with climbing image optimization using the same criteria, on the penalty potential with $\sigma$ of 10 .

Complete active space self-consistent field

Photoisomerization can proceed through crossings between potential energy surfaces, which requires electronic structure theory that can treat electronic degeneracies ${ }^{49}$. The CASSCF method is therefore a workhorse in the study of photoisomerization and photochemical reactions. Previous computations on 1-phenyl-1,3-butadiene have analyzed the potential energy surface with the SA2CAS $(10,10)$ SCF $/ 6-31+G^{*}$ level of theory, including the 10 carbon p orbitals involved in $\pi$-bonding ${ }^{22}$. These computations showed the excited state is more planar due to increased coupling between the 
1 phenyl and diene moiety, and a low energy $\left(3 \mathrm{kcal} \mathrm{mol}^{-1}\right.$ higher than the $\mathrm{S}_{1}$ minimum) conical

2 intersection was found leading to a spiro-cyclization photoproduct, which agrees with available

3 experimental data. In contrast, the isomerization pathways were found to be much higher at about 25

$4 \mathrm{kcal} \mathrm{mol}^{-1}$ above the $\mathrm{S}_{1}$ minimum. The isomerization MECI can be described as s-transoid (s-trans with

5 respect to the phenyl diene single bond), and a central MECI by analogy with butadiene ${ }^{19}$.

Butadiene has also been extensively studied and it has been found that SA-CASSCF with the nonintuitive active space of four electrons in three orbitals closely approximates both second order MS-CAS $(4,4) P T 2^{50}$ and experiment. This choice of active space destabilizes the $\mathrm{S}_{1}$ surface while

9 leaving the $S_{2}$ surface largely unchanged, restoring the near-degeneracy of $S_{2}$ and $S_{1}$ in the Franck10 Condon region. The transoid MECI is about $39 \mathrm{kcal} \mathrm{mol}^{-1}$ below the Franck Condon point, the $\mathrm{S}_{1}$ 11 minimum of butadiene is a twisted methylene. At the higher SA-3-(4,4)-MSPT2/6-31G** level of 12 theory ${ }^{21}$ the transoid MECI is about $22 \mathrm{kcal} \mathrm{mol}^{-1}$ below the planar $2{ }^{1} \mathrm{~A}_{\mathrm{g}}$ state, and $41.5 \mathrm{kcal} \mathrm{mol}^{-1}$ 13 below the Franck-Condon point.

The full $\pi$ space of cc-DPB requires a $(16,16)$ active space which is at the high-end of computational capabilities, especially for the relaxed reaction path optimization performed herein.

16 Furthermore, a larger active space is not always better, and this is perhaps evident in 1-phenyl-1,317 butadiene that has a large isomerization barrier at the SA2-CAS $(10,10)$ level. Therefore, for an initial 18 investigation it is reasonable to begin with a smaller active space. To our satisfaction, the SA319 CAS(4,3)SCF captures spectroscopic characteristics in agreement with SA3-(4,4,)-MS-PT2 20 calculations. The bright, ionic $\mathrm{S}_{1}$ state has $\mathrm{B}_{\mathrm{u}}$-like symmetry in agreement with experiment ${ }^{51}$, and, like $21 \mathrm{~PB}^{22}$, is described as bichromophoric (see Supporting Information). The dark, covalent $\mathrm{S}_{2}$ state has $22 \mathrm{~A}_{\mathrm{g}}$ symmetry and is approximately $1 \mathrm{eV}(23 \mathrm{kcal} / \mathrm{mol})$ above the $\mathrm{S}_{1}$ state. Experiments place the $\mathrm{S}_{2}$ state of tt-DPB $3.3 \mathrm{kcal} / \mathrm{mol}$ below the $1{ }^{1} \mathrm{~B}_{\mathrm{u}}$ state in the gas-phase, but the $1^{1} \mathrm{~B}_{\mathrm{u}} \rightarrow 1^{1} \mathrm{~A}_{\mathrm{g}}$ transition energy decreases with increasing medium polarizability; for example, $S_{1}$ is the $1^{1} B_{u}$ state in all hydrocarbon solvents ${ }^{51}$. Furthermore, these experiments on tt-DPB observe that the isomerization occurs via the $1^{1} \mathrm{~B}_{\mathrm{u}}$ state ${ }^{51}$. Note that this is different than butadiene where the covalent $2^{1} \mathrm{~A}_{\mathrm{g}}$ state is below the ionic $1^{1} \mathrm{~B}_{\mathrm{u}}$ state, and the trans/cis isomerization is preceded by passageway through the $1^{1}$ $\mathrm{B}_{\mathrm{u}} / 2^{1} \mathrm{~A}_{\mathrm{g}}\left(\mathrm{S}_{2} / \mathrm{S}_{1}\right)$ conical intersections ${ }^{50}$. Therefore, SA3-CAS $(4,3)$ SCF qualitatively reproduces key

30 PT2 calculations, it predicts $S_{2}$ as the bright state and the excitation is localized on the butadiene 31 chromophore. 
2 Results and Discussion

3 The analysis of cc-DPB photoisomerization pathways begins with a brief description of the

4 crystal and gas-phase ground- and excited-state structures. Next, single double-bond isomerization

5 mechanisms are revealed, with details about why these are prohibited in the crystal. Then a

6 comprehensive analysis of BP pathways is provided. Finally, the BP reaction paths for all conformers

7 within the unit crystal are compared to determine if certain conformations isomerize with faster rates

8 than others.

9 Crystal Description

Table 1 CASSCF excitation energies in $\mathrm{eV}$, (CASPT2 energies of the monomer in gas-phase)

\begin{tabular}{lcc}
\multicolumn{1}{c}{ Geometry } & $\Delta \mathrm{E}_{\text {vert }}$ & $\Delta \mathrm{E}_{\text {adiab }}$ \\
\hline A isomer & 5.942 & $4.468(3.794)$ \\
$\mathbf{B}$ isomer & 6.000 & $4.600(3.767)$ \\
C isomer & 5.827 & $4.250(3.562)$ \\
D isomer & 6.050 & $4.453(3.771)$ \\
Perpendicular & 5.727 & 4.317 \\
Vacuum & & 4.175 \\
Parallel Vacuum & $7.513^{*}$ &
\end{tabular}


The cc-DPB unit cell contains

2 two conformers of the monomer, for a

3 total of four DPB molecules. One of the

4 conformers has the phenyls in

5 perpendicular planes $(\mathbf{A}, \mathbf{B})$ and the

6 other has phenyls in parallel planes

7 (C,D), see Figure 3. The two conformers

8 of cc-DPB arrange in edge-to-face

9 alternating arrays: the perpendicular

10 conformers clasp together in a row of

11 the array, and the parallel conformers

12 clasp together in the next row of the

13 array. The parallel and perpendicular

14 conformers are also stable geometries in

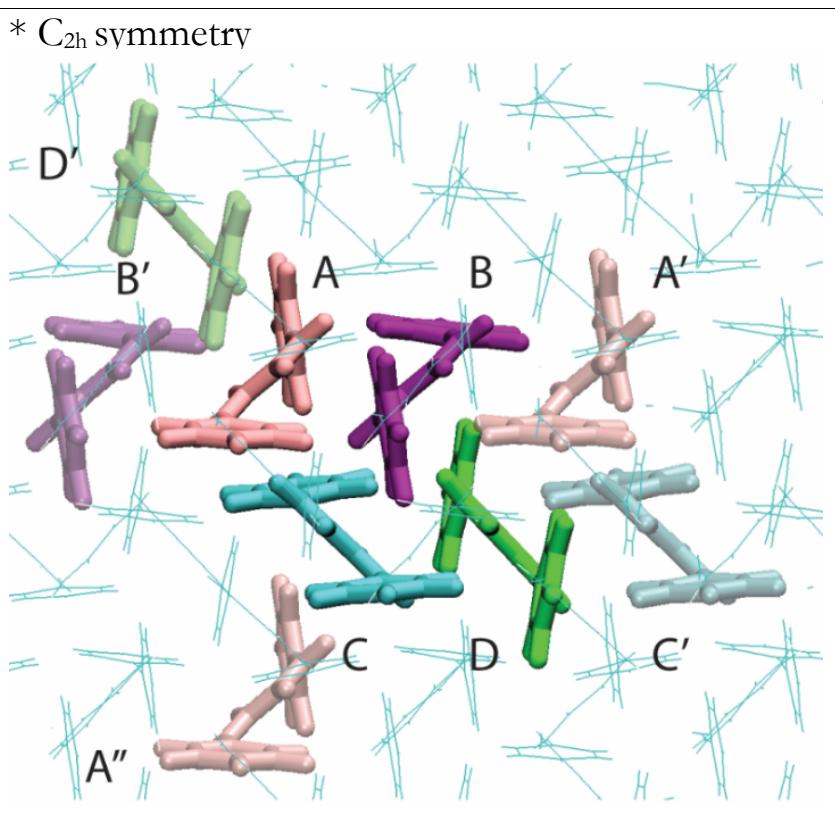

Figure 3. Unit cell with labels. Cyan: A, Purple: B, Red: C, Green D. The lighter colored monomers are members of adjacent cells, which interact with the central conformers.

15 the gas phase. In the gas phase the angle of the diene with respect to the phenyl planes is $90^{\circ}$, compared to the crystal angles of $\sim 45^{\circ}$.

The calculated vertical and adiabatic excitation energies of each of the conformers in the crystal phase and gas-phase are reported in Table 1. The experimental vertical and adiabatic excitation energies can be estimated from the excitation and emission spectrum, respectively, and are approximately $3.387 \mathrm{eV}(366 \mathrm{~nm})$ and $3.024 \mathrm{eV}(410 \mathrm{~nm})^{8}$. In contrast, in cyclohexane solution the vertical and adiabatic excitation energies are $4.27 \mathrm{eV}(290 \mathrm{~nm})$ and $3.56 \mathrm{eV}(360 \mathrm{~nm})^{51}$. Therefore, the

22 crystal exhibits a strong bathochromic shift with respect to solution, which is known to arise from the

23 long-range electrostatic effects in crystals ${ }^{32,33}$. The CASSCF results show significantly higher excitation

24 energies, due to the well-known lack of dynamical electron correlation. CASPT2, which includes

25 dynamical electron correlation, brings the energies closer in line with solution values, but does not 26 account for the environmental effects of the bulk crystal. 
OBF Double Bond Rotation

The photoisomerizations of conformer $\mathbf{C}$ were examined first because this conformer has the lowest energy initial excited state. Two cis,cis-s-transoid MECIs were found, where the s-trans 4 nomenclature is with respect to the central single bond. These MECI are labeled as cc-transoid-L and $5 \quad c c$-transoid-R because they resemble the rotation of the left and right side of a bicycle pedal, respectively 6 (top row Figure 4, and Table 2). To understand how the crystal steric environment affects these MECI, 7 the gas-phase MECIs were also found. The structures are overlaid to show how the crystal affects the 8 geometry, and the MECI energies are shown in the color matching the conformer (e.g. dark blue for 9 gas phase). The crystalline cc-transoid MECI differ from the gas phase, in that they are higher in energy 10 than their respective $S_{1}$ minimum by 6.6 and $13.1 \mathrm{kcal} \mathrm{mol}^{-1}$ for cc-transoid- $L$ and cc-transoid-R, 11 respectively ${ }^{52}$. The increase in energy of conical intersections from gas-phase to crystal can be 12 explained by restrictions on intramolecular rotation (RIR) $)^{53,54}$. As can be seen in Figure 4, the gas13 phase cc-transoid MECI are able to significantly deviate from planarity $\left(\alpha=43^{\circ}\right.$ and $\left.51^{\circ}\right)$ whereas the 14 crystalline phenyls require less than half the amount of out-of-plane rotation, consistent with RIR. 15 Rotating out of plane is energetically favorable in the gas phase because it helps the phenyl maintain 16 conjugation with the rotating interior hydrogen ( $\mathrm{H} 4$ or $\mathrm{H} 5)$. In contrast, $\alpha$ is prevented from changing 17 due to steric repulsion in the crystal.

Table 2 Single double-bond isomerization MECI energies and geometric characteristics.

\begin{tabular}{|c|c|c|c|c|c|c|}
\hline & & $\mathrm{S}_{0^{\mathrm{a}}}$ & $\mathrm{S}_{1^{\mathrm{a}}}$ & $\theta^{\mathrm{b}}$ & $\varphi^{\mathrm{b}}$ & $\alpha^{\mathrm{b}, \mathrm{c}}$ \\
\hline \multirow{2}{*}{ cc-transoid-L } & gas-phase & -1.8 & -1.7 & -83.6 & -130.9 & 42.8 \\
\hline & crystal-phase & 6.6 & 6.7 & -86.6 & -137.7 & 20.6 \\
\hline \multirow{2}{*}{ cc-transoid-R } & gas-phase & -1.8 & -1.7 & 85.3 & 131.4 & 50.6 \\
\hline & crystal-phase & 13.1 & 13.1 & 83.6 & 137 & 23.7 \\
\hline \multirow{2}{*}{ s-transoid } & gas-phase & -1.4 & -1.3 & -69.6 & -166.1 & 40.3 \\
\hline & crystal-phase & 3.0 & 3.2 & -29.5 & -174.4 & 3.8 \\
\hline \multirow{2}{*}{ s-central } & gas-phase & -7.0 & -6.9 & 115.6 & -173.1 & 120.8 \\
\hline & crystal-phase & -0.6 & -0.6 & 38.0 & 166.8 & 29.1 \\
\hline
\end{tabular}

\footnotetext{
a Energy in $\mathrm{kcal} / \mathrm{moll}$ with respect to the local $\mathrm{S}_{1}$-minimum.

$\mathrm{b}$ angle in degrees, see Figure 4

${ }^{c} \alpha$ is defined as the angle between the planes of the phenyl and the $\mathrm{S}_{1}$-minimum.
} 
The MECI geometries imply the existence of specific photoproducts. Herein the gas-phase $c c$ transoid are assumed to lead to OBF and HT-2 (Figure 5) because they contain a combination of 3 double-bond rotation $(\theta)$ and adjacent single-bond rotation $(\varphi)$ coordinates (Figure 4$)^{24}$. In the OBF 4 mechanism the phenyl completes the $\sim 180^{\circ}$ twist about the carbon-carbon double bond. For example, OBF characteristics can be seen in the angle $\alpha$ as the phenyl group flips over C3-C4 in Figure 4. In the HT mechanism single-bond rotation about the central double bond allows the CH group (e.g. C4H4) to complete the twist about the double bond without flipping the phenyl; $\alpha$ decreases as the hydrogen finishes the hula-twist. Interestingly, the perpendicular geometry $\alpha$ begins at $\sim 90^{\circ}$, therefore

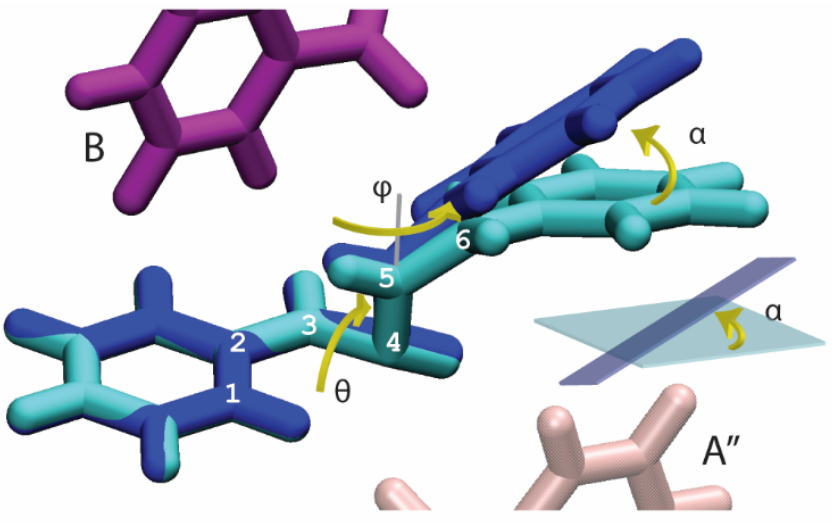

cc-transoid-L

S0: $6.6(-1.8) \mathrm{kcal} / \mathrm{mol}$

$\mathrm{S} 1: 6.7(-1.7) \mathrm{kcal} / \mathrm{mol}$

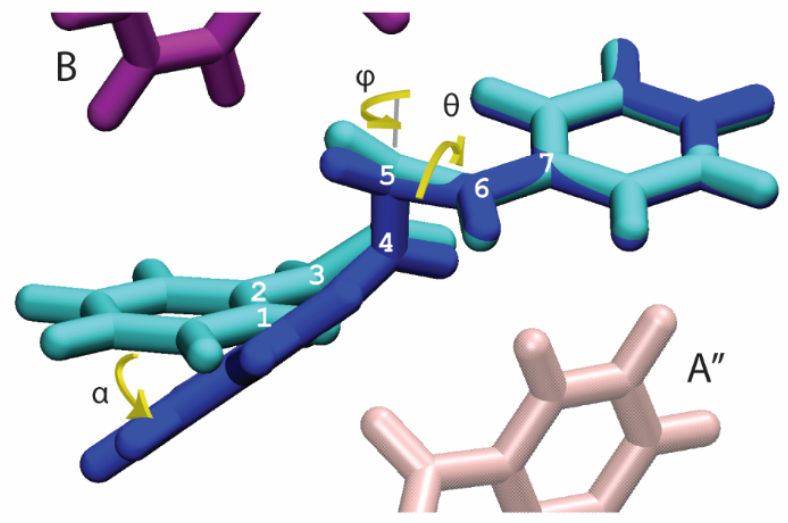

cc-transoid-R

S0: $13.1(-1.8) \mathrm{kcal} / \mathrm{mol}$

$\mathrm{S} 1: 13.1(-1.7) \mathrm{kcal} / \mathrm{mol}$

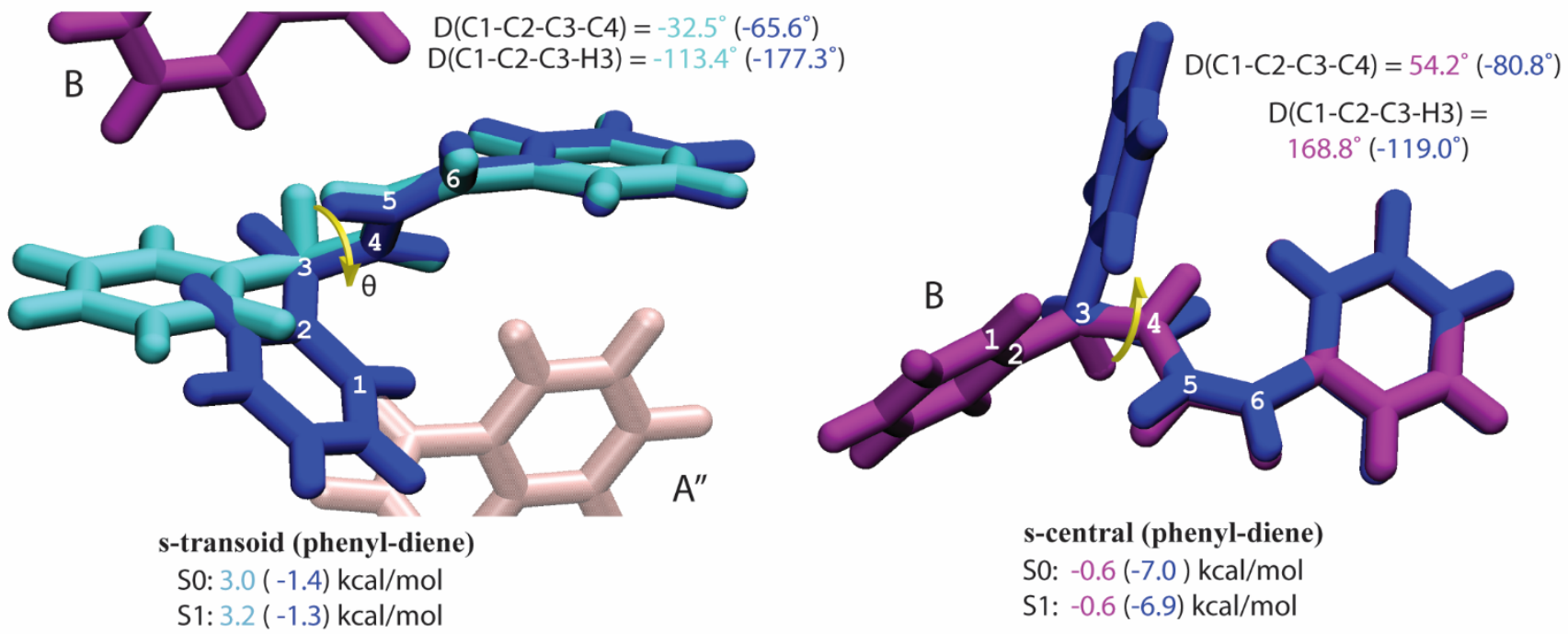

Figure 4. Geometries of gas-phase (dark blue) and crystalline transoid conical intersections (see Figure 3) involved in single double bond isomerization. The important coordinates are labeled one-bond flip coordinate $(\theta)$, single-bond rotation coordinate $(\varphi)$ and phenyl out-of-plane angle $(\alpha) . \alpha$ is defined as the angle between the planes of the phenyls of the MECI and $\mathrm{S}_{1}$ minimum. The energies and values for the different parameters are shown in the color matching the conformer: dark blue for gas-phase, cyan for $\mathbf{C}$, purple for $\mathbf{B}$. The $\mathrm{S}_{0}$ and $\mathrm{S}_{1}$ energies, in $\mathrm{kcal} \mathrm{mol}^{-1}$ are relative to the respective $\mathrm{S}_{1}$ minimum geometry. 
1 only half the necessary phenyl rotation is required for $\mathrm{OBF}$, which may facilitate OBF in the gas2 phase.

The expected photoproducts in the crystal-phase, however, are much different. The cc-transoid MECI distortions imply that completion of the OBF and HT are no longer allowed ${ }^{24}$. OBF is clearly 5 suppressed in the crystal state via restrictions on out-of-plane rotation due to the constrained cavity.

6 Furthermore, although $\alpha$ is smaller in the crystal phase, rotation about the central single bond $(\varphi)$ is

7 also suppressed in the crystal lattice, and this is evident in the MECI with $\varphi$ about $7^{\circ}$ larger; a smaller

$8 \varphi$ means greater rotation. This is interesting because it shows that although HT is more volume 9 conserving than $\mathrm{OBF}$ it still requires that the phenyls "slide" via the single-bond rotation coordinate $\varphi$, which is prohibited in the crystal phase.

Another class of CI important for single double-bond isomerization occurs via 12 pyramidalization at the first carbon of the diene. Two $\mathrm{CI}^{22}$ with pyramidalization at the first carbon 13 can be found in the gas-phase for cc-DPB. The two differ in the dihedral phenyl-diene single bond, 14 and can be described as s-central and s-transoid where the s-trans and s-central naming refers to the phenyl15 diene single bond (Figure 4 bottom row and Table 2) ${ }^{22}$. These CI are assumed to lead to HT-1 and 16 OBF photoproducts (Figure 5). The difference in photoproducts is the orientation of the resulting

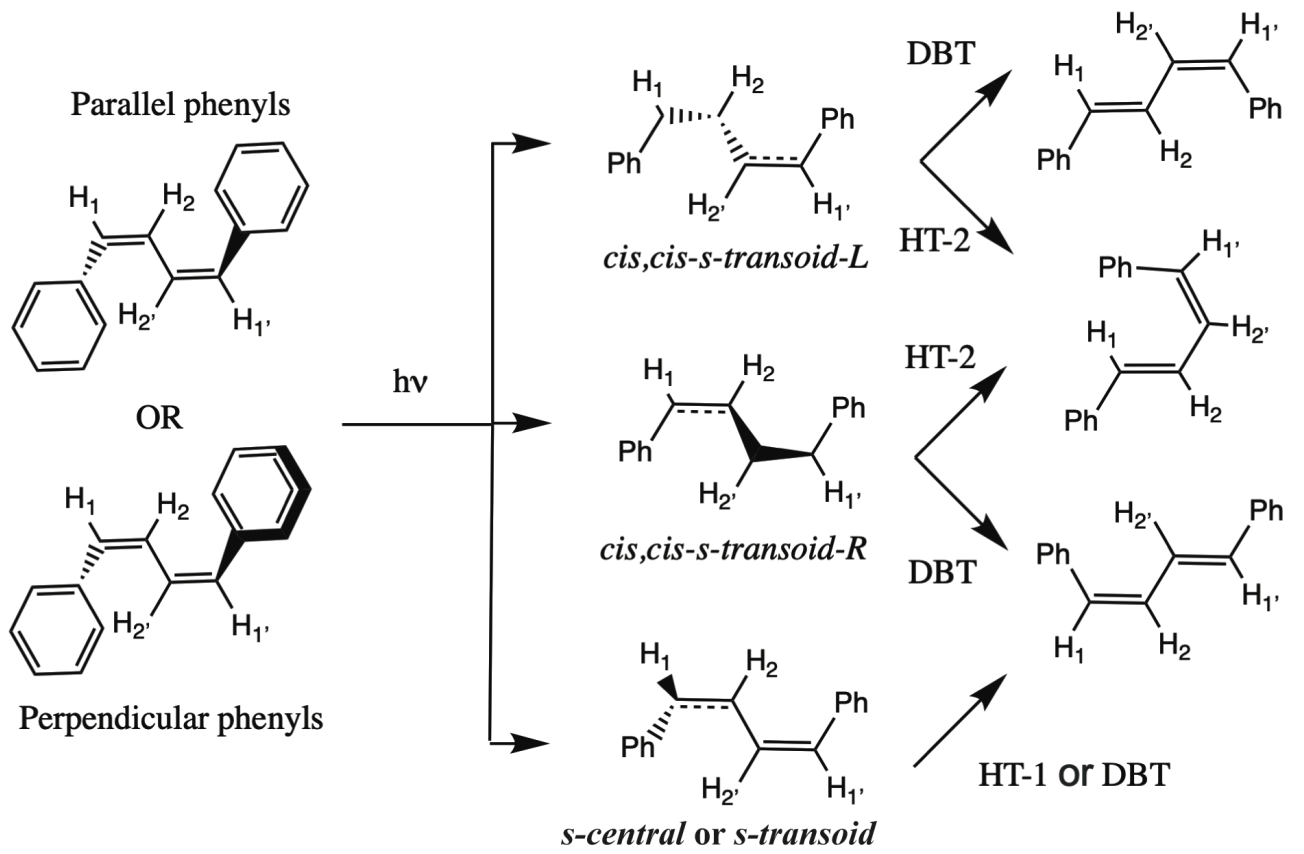

Figure 5. Single double-bond isomerization mechanisms. Operative only in the gas phase due to steric blockage in the crystal. 
1 phenyl: in $\mathrm{OBF}$ the phenyl ring flips $180^{\circ}$ about the phenyl-diene carbon-carbon single bond, whereas

2 HT-1 preserves the relative phenyl position.

Crystal phase planar analogues of $s$-transoid and $s$-central were found starting from the parallel phenyl geometries $(\mathbf{C})$ and perpendicular phenyl geometries $(\mathbf{B})$, respectively. In comparison to $c c$ transoid, an even greater difference between gas phase and crystal phase exists for this pair of conical intersections. In the crystal phase, the dominant motion rotates the hydrogen out of plane, e.g. D(C1-

7 C2-C3-H3) $=-113.4^{\circ}$ and the phenyls remain in plane (e.g. $\alpha=4^{\circ}$ ). This is unusual because rotation of 8 hydrogen out of plane has only been found in the gas-phase as a high-energy seam saddle point ${ }^{23,42,55}$. 9 In order for this conical intersection to become responsible for the HT in the condensed phase, the 10 steric surroundings must in some way cause this conical intersection to become lower energy. This 11 has previously been hypothesized ${ }^{23,42,55}$, but was not tested until now due to the lack of capacity to 12 simulate the reaction mechanism in the condensed phase, and therefore highlights the importance of 13 explicitly modeling the steric environments: the nature of the potential energy surface topology 14 including the seam space can change dramatically in the condensed phase. Incidentally, SE-GSM was 15 particularly beneficial here because the gas-phase and crystal phase structures were found from the same input reaction coordinates. In contrast, the optimization of these structures via traditional 17 techniques where the gas-phase structure is used as a guess for the reacting system of the crystal would 18 not work because the gas-phase structures do not fit within the crystal cavity. Regardless, the crystalline

19 s-transoid and s-central CI analogues are also non-productive in the crystal phase due to restrictions on 20 side-to-side rotations.

21 Bicycle Pedal Rotation

The CI responsible for bicycle pedal isomerization in the crystal phase is now identified and analyzed. As demonstrated above, the cc-transoid-L and cc-transoid-R MECI each resemble one half24 rotation of a bicycle pedal. Putting these motions together in a concerted manner completes the cycle. 25 As can be seen in Figure 6a, the bicycle-pedal CI be described as trans,trans-s-transoid, or tt-transoid for 26 short. As above, the gas-phase tt-transoid is more non-planar than the crystalline form, and this extra 27 flexibility allows gas-phase $t$-transoid to reach a lower energy than the crystal phase. 
Excited-state reaction paths were found

2 connecting the $\mathrm{S}_{1}$-minimum and the tt-transoid

3 CI. In the crystal state, the reaction path is a

4 single, smooth elementary step consisting of

$5 \sim 180$ degree rotation of the central diene,

6 followed by pyramidalization (Figure 6b). In

7 contrast, in the gas-phase the reaction path

8 from the $\mathrm{S}_{1}$-minimum to tt-transoid undergoes

9 significant out-of-plane distortion as the

10 phenyls attempt to maintain conjugation with

11 the central rotating diene (see Supporting

12 Information). No exact transition state

13 geometry could be optimized, probably due to

14 the unstable (high energy) nature of the reaction

15 path. Taken together with the steepness of the

16 potential energy surface towards the cc-transoid

17 CI in the gas-phase (Figure 4), this indicates that

18 the BP pathway is not operable in the gas phase.

19 The crystal BP mechanism is also considerably

20 different than the solution-phase cc-DPB $\rightarrow \mathrm{tt}-$

21 DPB mechanism which proceeds through a cis-

22 phenylallylbenzyl excited state intermediate ${ }^{17}$.

23 For example the cis-phenylallylbenzyl

24 intermediate would require side-to-side motion

25 of the phenyl, which is not allowed in the

26 crystal. Therefore, the crystal state is unique in

27 its operation: the steric environment opens the

28 bicycle pedal isomerization reaction valley by

29 restricting out-of-plane motion and allowing

30 smooth rotation of the diene.
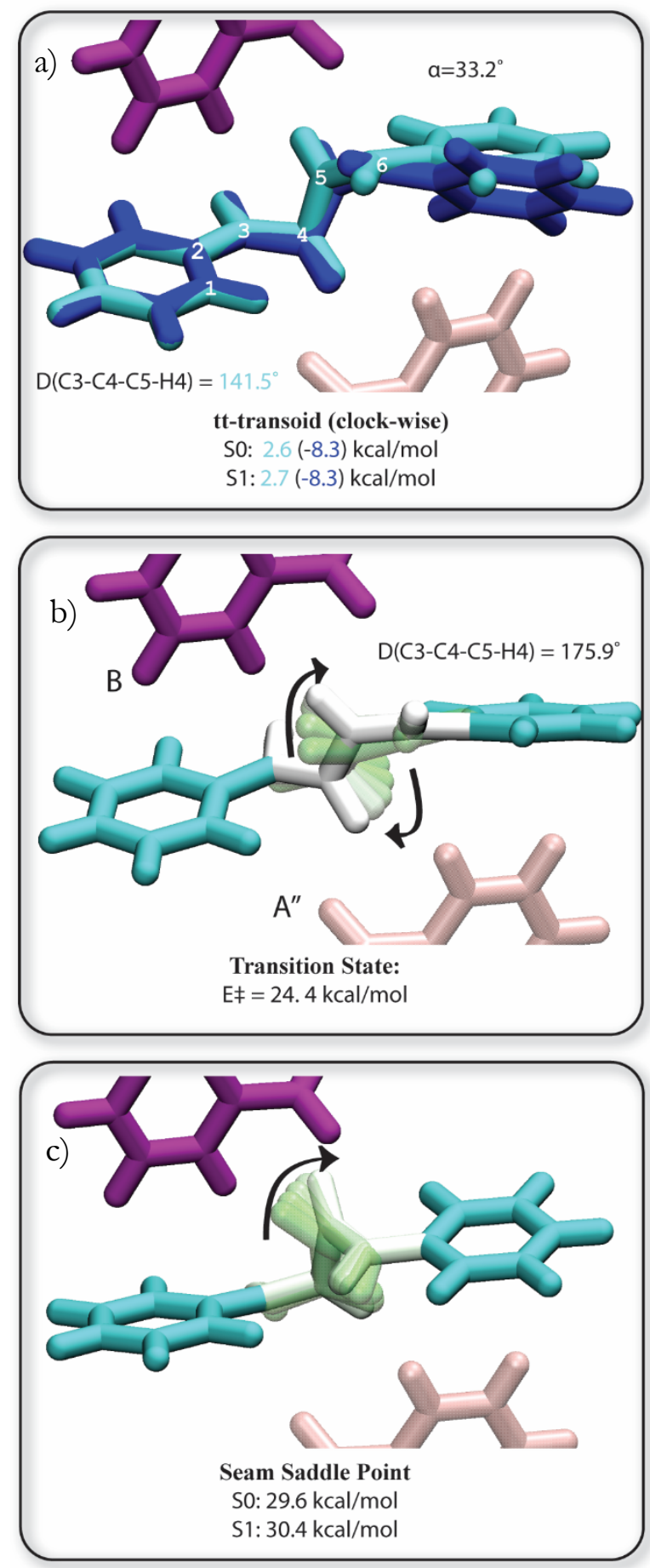

Figure 6. Relevant geometries for the bicycle pedal isomerization a) comparison of gas and crystal phase tt-transoid MECI, b) excitedstate transition state connecting $\mathrm{S}_{1}$ minimum and transoid MECI c) seam saddle point connecting cc-transoid- $\mathrm{R}$ and tt-transoid MECI. The $\mathrm{S}_{0}$ and $\mathrm{S}_{1}$ energies, in $\mathrm{kcal} \mathrm{mol}^{-1}$ are relative to the $\mathrm{S}_{1}$ minimum geometry of $\mathbf{C}$. 
Close inspection of the transition state geometry reveals additional important details. First, as

2 can be seen in Figure 6b, the pedal rotates neatly through the phenyl-diene gaps of the adjacent $\mathbf{B}$ and

3 A" molecules. The adjacent phenyls prevent out-of-plane rotation by locking the pedals into gear.

4 Thus, the BP isomerization resembles the mechanical operation of real bicycles in more than one way:

5 the concerted rotation, and the mechanical rigidity in being locked into this rotation pathway. Second,

6 the transition state geometry has no pyramidalization $\left(\mathrm{D}(\mathrm{C} 1-\mathrm{C} 2-\mathrm{C} 3-\mathrm{H} 2)=175.9^{\circ}\right)$ which indicates that

7 the isomerization might bifurcate to tt-DPB in the excited-state. Indeed, an excited-state reaction

8 pathway was found that connects the $S_{1}$-minimum and tt-DPB $S_{1}$-minimum with an identical

9 transition state geometry as Figure 6b, although they were computed independently. Therefore, this

10 shows that the transition state bifurcates to lead to the tt-DPB $\mathrm{S}_{1}$-minimum and $t$-transoid MECI. At

11 the $\mathrm{S}_{1}$-minima, the excited-molecule can decay non-radiatively at the tt-transoid through

12 pyramidalization, or radiatively through fluorescence.

13 MECI, however, are not enough to fully describe the photochemistry because non-radiative 14 decay can occur at any point along the high-dimensional seam-space. In particular, regions of the seam 15 are often found parallel to the adiabatic reaction coordinate and can contribute via motion orthogonal 16 to the reaction coordinate ${ }^{56}$. Therefore, to explore the potential energy surface more widely for other 17 possible bicycle pedal mechanisms, a minimum energy seam pathway was found connecting cc-transoid 18 and tt-transoid. This seam runs roughly parallel to the adiabatic pathway and the seam saddle point 19 occurs near the excited-state transition state (Figure 6c). Non-radiative decay through CI points before 20 the seam saddle point are considered to return to cc-DPB, whereas all CI points at or after the saddle 21 point lead to tt-DPB. With this seam established a complete picture of the relevant BP potential energy 22 surface is now known and a summary of the important pathways, productive and non-productive, is 23 given in Figure 7. 


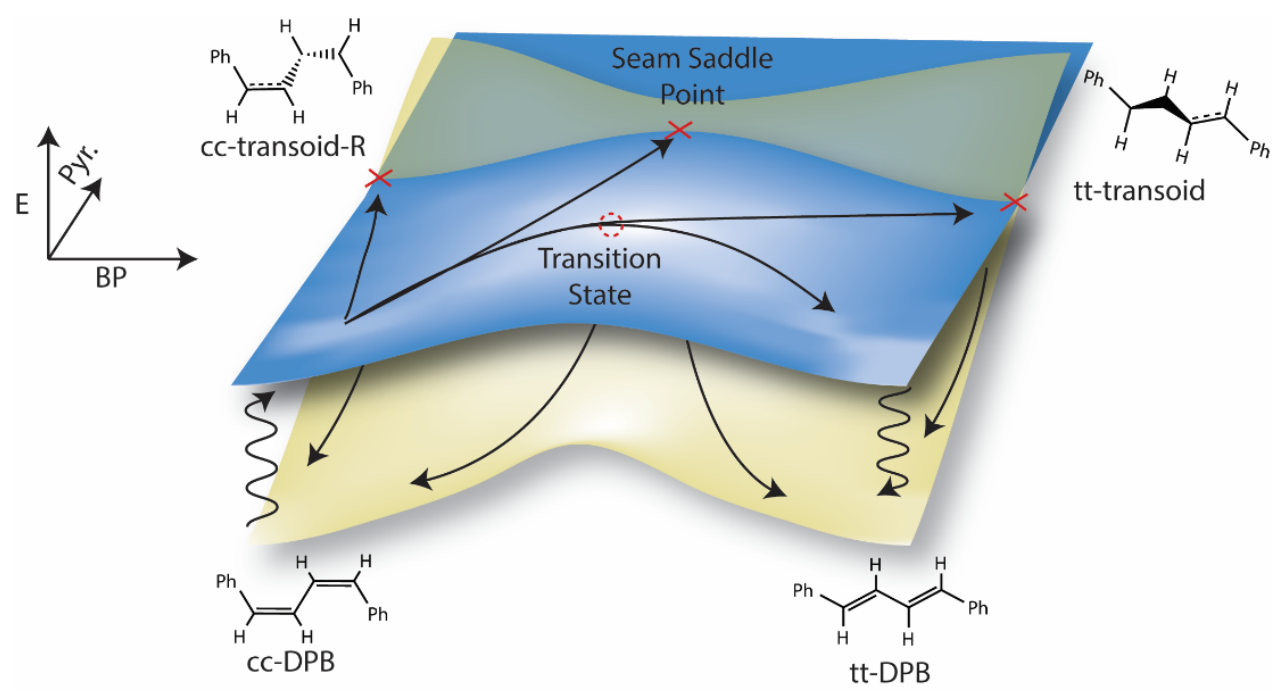

Figure 7. Summary of major excited-state reaction channels of conformer C, including productive and non-productive routes.

Figure 7 provides a summary of the proposed photoisomerization pathways. Starting from

2 photoexcited cc-DPB conformer $\mathbf{C}$, the transition state energy leading to the bicycle pedal CI (24.4

$\left.3 \mathrm{kcal} \mathrm{mol}^{-1}\right)$ is below the vertical excitation energy $\left(36.3 \mathrm{kcal} \mathrm{mol}^{-1}\right)$ suggesting that enough energy is

4 available to overcome the barrier (at least at short timeframes following excitation). This pathway is

5 much lower in energy than the ground-state process $\left(98.4 \mathrm{kcal} \mathrm{mol}^{-1}\right)$. However, a significant region

6 of the seam runs roughly parallel to the adiabatic coordinate, which can drain energy from the excited-

7 state via non-productive decay to the reactants (i.e. an aborted OBF or HT). The productive formation

8 of tt-DPB would only start around the seam-saddle point connecting $c$-transoid and $t$-transoid which is

9 much higher in energy. Nevertheless, despite the high-energy excited-state activation barrier the BP

10 isomerization pathways appears reasonable. If the phenyls are held stationary the motion would have

11 to be confined to the diene portion of the molecule. Furthermore, the gaps between the phenyls and

12 diene enable unimpeded passage for $\mathrm{CH}$ rotation. Therefore, at present the large barrier is considered

13 an artifact of the lack of quantitative energies provided by CASSCF (e.g. Error! Reference source

14 not found.) in computing these electronic states, or long-range interactions which are not captured in 15 the MM model. Future studies will test the accuracy of this relatively simple level of theory against 16 higher-accuracy methods. 
Now that the mechanism for cistrans photoisomerization in cc-DPB have

4 been outlined, the various conformers

5 within the unit cell are studied. Besides

6 categorization into parallel and

7 perpendicular units, the steric environment

8 surrounding the central double bond of

9 each conformer is different and could result

10 in different activation barriers for BP

11 isomerization. If the conformations have

12 different activation barriers, this could help

13 explain the experimentally observed

14 periods of fast and slow conversion

15 because certain conformers will isomerize

16 with a slower rate, and therefore,

17 presumably, with less photon efficiency ${ }^{36}$.

As we have seen, the primary

19 motion during the BP isomerization is the

20 rotation of the central $\mathrm{CH}$ groups between

21 the phenyl-diene gap of neighboring units.

22 The steric interaction of these "pedals" and

23 the neighboring monomers are therefore of

24 concern. Isomerization in the clockwise

25 direction versus the counter-clockwise

26 direction can also lead to differences in

27 isomerization efficiencies, depending on

28 conformer. To familiarize oneself with the

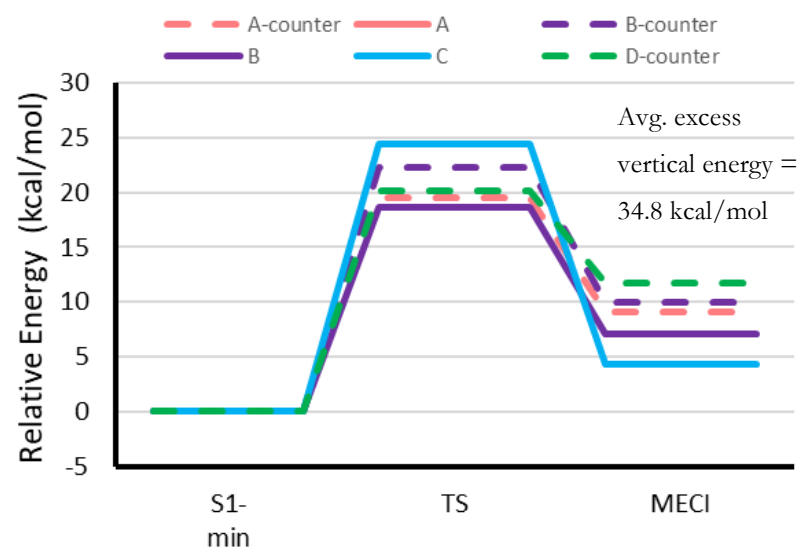

Figure 8. Comparison of bicycle pedal reaction paths to MECI for all conformers. The average vertical excess energy is over conformers A-D which have different microenvironments and energies.

29 different steric environments, consider the counter-clockwise rotations of $\mathbf{A}$ and $\mathbf{B}$ as an example.

30 The diene of unit $\mathbf{A}$ and $\mathbf{B}$ are oriented similarly, however the steric interactions upon isomerization

31 are different. The top pedal $(\mathrm{CH})$ of $\mathbf{A}$ slips between the gap of the phenyl and diene of $\mathbf{D}$ ' and the 
1 bottom pedal becomes perpendicular with the phenyl of $\mathbf{C}$ and rests near $\mathbf{B}$. On the other hand, the

2 bottom pedal of $\mathbf{B}$ slips between the phenyl diene gap of $\mathbf{D}$ and the top pedal sterically clashes with

3 A. Similar analysis has been performed for all conformers in both rotation directions, and is present 4 in Table 3. minimum of each conformer and the appropriate tt-transoid-CI were found for clockwise and counterclockwise rotations (Figure 8). A range of about $6 \mathrm{kcal} / \mathrm{mol}$ is observed between all conformers: conformer $\mathbf{B}$ has the lowest energy barrier of $18.6 \mathrm{kcal} / \mathrm{mol}$ in the clockwise direction, and conformer

$9 \mathbf{C}$ has the largest barrier of $24.5 \mathrm{kcal} / \mathrm{mol}$. Clockwise BP rotation of conformer $\mathbf{B}$ causes the top pedal 10 to pass first through the phenyl-diene gap of $\mathbf{A}^{\mathbf{\prime}}$ and then of $\mathbf{D}$. The bottom pedal of conformer $\mathbf{B}$ 11 skirts past $\mathbf{A}$ and does not pass directly through a phenyl-diene gap. In contrast when $\mathbf{B}$ rotates in the 12 counter-clockwise direction, the bottom pedal rotates through $\mathbf{D}$ and as it approaches $\mathbf{A}^{\mathbf{\prime}}$ it relaxes 13 before going through the gap. The top pedal of conformation $\mathbf{C}$ also does not pass through the phenyl 14 diene gap of $\mathbf{B}$ during clockwise rotation. Overall, however, these differences appear to be well within 15 errors of the current semi-quantitative model. For all MECI and RP diagrams of these various 16 conformers, consult the Supporting Information.

17 Nevertheless, to answer the question of whether the different conformations are responsible 18 for different isomerization rates in the experiments (fast and then slow), the answer is most likely 19 "no". All conformers can rotate without major impediment and the major steric interaction provided 20 by the crystal lattice is to restrict out-of-plane rotations of the phenyl, which is present in all 21 conformers. Furthermore, additional experiments have observed that the related compound cis,trans22 1,4-di-o-tolyl-1,3-butadiene (cc-DTB) has no conformational anisotropy ${ }^{11}$ yet also displays periods of 23 fast and slow isomerization kinetics. As a result, it is reasonable to suggest that conformational 24 differences are not responsible for the observed stepwise reaction rate, but instead the effect is due to more complex changes as the crystal reorganizes. 
The present computational investigation of cc-DPB provided substantial insight into the diverse OBF, HT, and BP mechanisms in the gas-phase and crystal states. The out-of-plane angle $\alpha$ and the side-to-side rotation angle $\varphi$ were found to be useful parameters to explain why OBF and HT were prohibited in the crystal phase. Interestingly, the crystal phase can fundamentally change the seam topology, as seen in how the HT-1 conical intersection in the crystal phase resembles gas-phase seam saddle points. The dramatic influence of the steric environment is also evident in the BP isomerization, where the phenyls are locked in place and the diene must rotate in a smooth volumeconserving manner. The specific way the monomers clasp together is also important for ensuring unimpeded passageway for the rotating diene. In contrast, cc-DPB is not able to isomerize via the BP

11 in the gas phase because it lacks a rigid structure that is essential for ensuring a smooth rotation, 12 without relaxation into non-BP geometries.

BP isomerization paths for unit-cell conformers revealed that all conformers isomerize in a 14 similar fashion. This indicates that the fast and slow reaction kinetics observed in experiment are not 15 likely to be a result of the different microenvironments in the unit cell, at least in the early stages of 16 isomerization before the crystal reorganizes.

The growing string method was instrumental in locating the reaction pathways, conical intersections, and seam spaces throughout this study. In particular, the qualitative differences in

19 isomerization pathways between the gas phase and solid state were straightforwardly delineated by 20 GSM, allowing these mechanisms to be brought to light without undue reliance on prior chemical 21 intuition. However, quantitative accuracy in describing the bicycle pedal has not yet been achieved. 22 Therefore, in addition to providing relevant details of the BP mechanism we hope that this study can 23 further motivate computational analysis of photochemical reactions in the solid state.

We are thankful to Professor Jack Saltiel for providing useful feedback on early drafts of this manuscript. This work is supported by NSF (CHE 1551994 to PZ) and Office of Naval Research (ONR-N0014-18-1-2659 to TJM). 
Supporting Information

MM parameters, molecular orbital diagrams, and reaction path profiles are provided in the Supporting

Information.

References

Liu, R. S. H.; Yang, L.-Y.; Liu, J. Mechanisms of Photoisomerization of Polyenes in Confined Media. From Organic Glasses to Protein Binding Cavities. Photochem. Photobiol. 2006, 2-10. https://doi.org/10.1562/2006-01-27-RA-786.

(2) Gerwien, A.; Schildhauer, M.; Thumser, S.; Mayer, P.; Dube, H. Direct Evidence for Hula Twist and Single-Bond Rotation Photoproducts. Nat. Commun. 2018, 9 (1), 1-9. https://doi.org/10.1038/s41467-018-04928-9.

(3) Redwood, C.; Bayda, M.; Saltiel, J. Photoisomerization of Pre- and Provitamin D3 in EPA at 77 K: One-Bond-Twist, Not Hula-Twist. J. Phys. Chem. Lett. 2013, 4 (5), 716-721. https://doi.org/10.1021/jz302108c.

(4) Fuß, W.; Kosmidis, C.; Schmid, W. E.; Trushin, S. A. The Photochemical Cis-Trans Isomerization of Free Stilbene Molecules Follows a Hula-Twist Pathway. Angew. Chemie - Int. Ed. 2004, 43 (32), 4178-4182. https://doi.org/10.1002/anie.200454221.

(5) Liu, R. S. H.; Hammond, G. S. The Case of Medium-Dependent Dual Mechanisms for Photoisomerization: One-Bond-Flip and Hula-Twist. Proc. Natl. Acad. Sci. 2000, 97 (21), 11153-11158. https://doi.org/10.1073/pnas.210323197.

(6) Fuß, W. Previtamin D: Z-E Photoisomerization via a Hula-Twist Conical Intersection. Phys. Chem. Chem. Phys. 2019, 21 (13), 6776-6789. https://doi.org/10.1039/c9cp00500e.

(7) Saltiel, J.; Krishna, T. S. R.; Clark, R. J. Photoisomerization of Cis,Cis-1,4-Diphenyl-1,3Butadiene in the Solid State: The Bicycle-Pedal Mechanism. J. Phys. Chem. A 2006, 110 (5), 1694-1697. https://doi.org/10.1021/jp056700d.

(8) Saltiel, J.; Krishna, T. S. R.; Laohhasurayotin, S.; Fort, K.; Clark, R. J. Photoisomerization of Cis , Cis - to Trans, Trans -1,4-Diaryl-1,3-Butadienes in the Solid State: The Bicycle-Pedal Mechanism. J. Phys. Chem. A 2008, 112 (2), 199-209. https://doi.org/10.1021/jp077342c.

(9) Furukawa, D.; Kobatake, S.; Matsumoto, A. Direct Observation of Change in the Molecular Structure of Benzyl (Z,Z)-Muconate during Photoisomerization in the Solid State. Chem. Commun. 2008, No. 1, 55-57. https://doi.org/10.1039/b714792a.

(10) Odani, T.; Matsumoto, A.; Sada, K.; Miyata, M. One-Way EZ-Isomerization of Bis(nButylammonium) (Z,Z)-Muconate under Photoirradiation in the Crystalline State. Chem. Commun. 2001, 19 (c), 2004-2005. https://doi.org/10.1039/b106155k.

(11) Saltiel, J.; Bremer, M. A.; Laohhasurayotin, S.; Krishna, T. S. R. Photoisomerization of Cis, Cisand Cis,Trans-1,4-Di-o-Tolyl-1,3-Butadiene in Glassy Media at $77 \mathrm{~K}$ : One-Bond-Twist and Bicycle-Pedal Mechanisms. Angew. Chemie Int. Ed. 2008, 47 (7), 1237-1240. https://doi.org/10.1002/anie.200704465.

(12) Tong, F.; Al-Haidar, M.; Zhu, L.; Al-Kaysi, R. O.; Bardeen, C. J. Photoinduced Peeling of Molecular Crystals. Chem. Commun. 2019, 55 (26), 3709-3712. https://doi.org/10.1039/c8cc10051a.

(13) Sonoda, Y.; Kawanishi, Y.; Tsuzuki, S.; Goto, M. Crystalline-State Z,E-Photoisomerization of a Series of (Z,E,Z)-1,6-Diphenylhexa-1,3,5-Triene 4,4'-Dicarboxylic Acid Dialkyl Esters. Chain 
Length Effects on the Crystal Structure and Photoreactivity. J. Org. Chem. 2005, 70 (24), 97559763. https://doi.org/10.1021/jo051137g.

(14) Saltiel, J.; Redwood, C. E.; Laohhasurayotin, K.; Samudrala, R. Photochemistry of the 1,6Dideuterio-1,3,5-Hexatrienes in Solution: Efficient Terminal Bond Photoisomerization in One-Bond-Twist and Bicycle Pedal Ways. J. Phys. Chem. A 2018, 122 (43), 8477-8489. https://doi.org/10.1021/acs.jpca.8b08288.

(15) Jung, Y. O.; Lee, J. H.; Kim, J.; Schmidt, M.; Moffat, K.; Šrajer, V.; Ihee, H. Volume-Conserving Trans-cis Isomerization Pathways in Photoactive Yellow Protein Visualized by Picosecond XRay Crystallography. Nat. Chem. 2013, 5 (3), 212-220. https://doi.org/10.1038/nchem.1565.

(16) A, W. Bicycle-Pedal Model for First Step in Vision Process. Nature 1976, 260, 679.

(17) Saltiel, J.; Redwood, C. E. Photochemistry of the 1,4-Diphenyl-1,3-Butadienes in Ethanol. Trapping Conical Intersections. J. Phys. Chem. A 2016, 120 (18), 2832-2840. https://doi.org/10.1021/acs.jpca.6b02330.

(18) Saltiel, J.; Bremer, M. A.; Laohhasurayotin, S.; Krishna, T. S. R. Photoisomerization of Cis,Cisand Cis,Trans-1,4-Di-o-Tolyl-1,3-Butadiene in Glassy Media at $77 \mathrm{~K}$ : One-Bond-Twist and Bicycle-Pedal Mechanisms. Angew. Chemie - Int. Ed. 2008, 47 (7), 1237-1240. https://doi.org/10.1002/anie.200704465.

(19) Olivucci, M.; Ragazos, I. N.; Bernardi, F.; Robb, M. A. A Conical Intersection Mechanism for the Photochemistry of Butadiene. A MC-SCF Study. J. Am. Chem. Soc. 1993, 115 (9), 37103721. https://doi.org/10.1021/ja00062a042.

(20) Kuhlman, T. S.; Glover, W. J.; Mori, T.; Moller, K. B.; Martínez, T. J. Between Ethylene and Polyenes - The Non-Adiabatic Dynamics of Cis-Dienes. Faraday Discuss. 2012, 157, 193-212. https://doi.org/10.1039/c2fd20055d.

(21) Glover, W. J.; Mori, T.; Schuurman, M. S.; Boguslavskiy, A. E.; Schalk, O.; Stolow, A.; Martínez, T. J. Excited State Non-Adiabatic Dynamics of the Smallest Polyene, Trans 1,3-Butadiene. II. $\mathrm{Ab}$ Initio Multiple Spawning Simulations. J. Chem. Phys. 2018, 148 (16). https://doi.org/10.1063/1.5018130.

(22) Ogliaro, F.; Wilsey, S.; Bearpark, M. J.; Sardo-Infirri, S. Interpreting the Excited States and Decay Processes of Bichromophoric 1-Phenyl-1,3-Butadiene Using CASSCF Calculations. Mol. Phys. 2006, 104 (5-7), 1017-1032. https:/ / doi.org/10.1080/00268970500418307.

(23) Norton, J. E.; Houk, K. N. H/Vinyl Conical Intersections of Hexatrienes Related to the HulaTwist Photoisomerization. Mol. Phys. 2006, $104 \quad$ (5-7), 993-1008. https://doi.org/10.1080/00268970500417606.

(24) Sampedro Ruiz, D.; Cembran, A.; Garavelli, M.; Olivucci, M.; Fuß, W. Structure of the Conical Intersections Driving the Cis-trans Photoisomerization of Conjugated Molecules. Photochem. Photobiol. 2002, $76 \quad$ (6), 622. https://doi.org/10.1562/00318655(2002)076<0622:SOTCID>2.0.CO;2.

(25) Wilsey, S.; Houk, K. N. H-Vinyl Conical Intersections for Dienes: A Mechanism for the Photochemical Hula Twist. Photochem. Photobiol. 2002, 76 (6), 616-621. https://doi.org/10.1562/0031-8655(2002)076<0616:HVCIFD>2.0.CO;2.

(26) Zimmerman, H. E.; Sebek, P.; Zhu, Z. Ab Initio Computations of Reacting Species in Crystal Lattices; Mechanistic and Exploratory Organic Photochemistry [17]. Journal of the American Chemical Society. 1998, pp 8549-8550. https://doi.org/10.1021/ja980042b.

(27) Zimmerman, H. E.; Alabugin, I. V; Smolenskaya, V. N. Experimental and Theoretical HostGuest Photochemistry; Control of Reactivity with Host Variation and Theoretical Treatment with a Stress Shaped Reaction Cavity; Mechanistic and Exploratory Organic Photochemistry. Tetrahedron 2000, 56 (36), 6821-6831. https://doi.org/10.1016/S0040-4020(00)00504-4.

(28) Zimmerman, H. E.; Nesterov, E. E. Development of Experimental and Theoretical Crystal 
Lattice Organic Photochemistry: The Quantitative Cavity . Mechanistic and Exploratory Organic. 2002, 35 (2), 77-85. https://doi.org/10.1021/ar000210g.

(29) Ruiz-Barragan, S.; Morokuma, K.; Blancafort, L. Conical Intersection Optimization Using Composed Steps Inside the ONIOM(QM:MM) Scheme: CASSCF:UFF Implementation with Microiterations. J. Chem. Theory Comput. 2015, 11 (4), 1585-1594. https://doi.org/10.1021/acs.jctc.5b00004.

(30) Bučko, T.; Hafner, J.; Lebègue, S.; Ángyán, J. G. Spin Crossover Transition of Fe(Phen)2(NCS)2: Periodic Dispersion-Corrected Density-Functional Study. Phys. Chem. Chem. Phys. 2012, 14 (16), 5389-5396. https://doi.org/10.1039/c2cp40111h.

(31) Presti, D.; Labat, F.; Pedone, A.; Frisch, M. J.; Hratchian, H. P.; Ciofini, I.; Cristina Menziani, M.; Adamo, C. Modeling Emission Features of Salicylidene Aniline Molecular Crystals: A QM/QM' Approach. J. Comput. Chem. 2016, 37 (9), 861-870. https://doi.org/10.1002/jcc.24282.

(32) Wilbraham, L.; Adamo, C.; Labat, F.; Ciofini, I. Electrostatic Embedding to Model the Impact of Environment on Photophysical Properties of Molecular Crystals: A Self-Consistent Charge Adjustment Procedure. J. Chem. Theory Comput. 2016, 12 (7), 3316-3324. https://doi.org/10.1021/acs.jctc.6b00263.

(33) Rivera, M.; Dommett, M.; Crespo-Otero, R. ONIOM(QM:QM') Electrostatic Embedding Schemes for Photochemistry in Molecular Crystals. J. Chem. Theory Comput. 2019, 15 (4), 2504 2516. https://doi.org/10.1021/acs.jctc.8b01180.

(34) Aldaz, C.; Wang, L.-P.; Martinez, T. J.; Zimmerman, P. M. A Linear Scaling Growing String Method with Correlated Motions, In Preparation. 2020.

(35) Wang, L. P.; Song, C. Geometry Optimization Made Simple with Translation and Rotation Coordinates. J. Chem. Phys. 2016, 144 (21), 214108. https://doi.org/10.1063/1.4952956.

(36) Zimmerman, H. E.; Nesterov, E. E. Crystal Lattice Photochemistry Often Proceeds in Discrete Stages. Mechanistic and Exploratory Organic Photochemistry 1, 2. Org. Lett. 2000, 2 (8), 1169_ 1171. https://doi.org/10.1021/ol0057838.

(37) Zimmerman, H. E.; Alabugin, I. V; Chen, W.; Zhu, Z.; June, R. V. Dramatic Effects of Crystal Morphology on Solid State Reaction Course; Control by Crystal Disorder; Mechanistic and Exploratory Organic Photochemistry. J. Am. Chem. Soc. 1999, 121 (6), 11930-11931. https://doi.org/10.1021/ja992208u.

(38) Aldaz, C. https://github.com/ZimmermanGroup/pyGSM.

(39) Zimmerman, P. M. Growing String Method with Interpolation and Optimization in Internal Coordinates: Method and Examples. J. Chem. Phys. 2013, 138 (18), 1-11. https://doi.org/10.1063/1.4804162.

(40) Zimmerman, P. M. Single-Ended Transition State Finding with the Growing String Method. J. Comput. Chem. 2015, 36 (9), 601-611. https://doi.org/10.1002/jcc.23833.

(41) Dewyer, A. L.; Zimmerman, P. M. Finding Reaction Mechanisms, Intuitive or Otherwise. Org. Biomol. Chem. 2017, 501-504. https://doi.org/10.1039/C6OB02183B.

(42) Aldaz, C.; Kammeraad, J. A.; Zimmerman, P. M. Discovery of Conical Intersection Mediated Photochemistry with Growing String Methods. Phys. Chem. Chem. Phys. 2018, 20 (43), 27394 27405. https://doi.org/10.1039/C8CP04703K.

(43) Baker, J.; Kessi, A.; Delley, B. The Generation and Use of Delocalized Internal Coordinates in Geometry Optimization. J. Chem. Phys. 1996, 105 (1996), 192-212. https://doi.org/10.1063/1.471864.

(44) Ufimtsev, I. S.; Martinez, T. J. Quantum Chemistry on Graphical Processing Units. 3. Analytical Energy Gradients, Geometry Optimization, and First Principles Molecular Dynamics. J. Chem. 
Theory Comput. 2009, 5 (10), 2619-2628. https://doi.org/10.1021/ct9003004.

(45) Hohenstein, E. G.; Luehr, N.; Ufimtsev, I. S.; Martínez, T. J. An Atomic Orbital-Based Formulation of the Complete Active Space Self-Consistent Field Method on Graphical Processing Units. J. Chem. Phys. 2015, 142 (22). https://doi.org/10.1063/1.4921956.

(46) Isborn, C. M.; Götz, A. W.; Clark, M. A.; Walker, R. C.; Martínez, T. J. Electronic Absorption Spectra from MM and Ab Initio QM/MM Molecular Dynamics: Environmental Effects on the Absorption Spectrum of Photoactive Yellow Protein. J. Chem. Theory Comput. 2012, 8 (12), 50925106. https://doi.org/10.1021/ct3006826.

(47) Eastman, P.; Swails, J.; Chodera, J. D.; McGibbon, R. T.; Zhao, Y.; Beauchamp, K. A.; Wang, L. P.; Simmonett, A. C.; Harrigan, M. P.; Stern, C. D.; Wiewiora, R. P.; Brooks, B. R.; Pande, V. S. OpenMM 7: Rapid Development of High Performance Algorithms for Molecular $\begin{array}{llllll}\text { Dynamics. } & \text { PLoS Comput. Biol. } & \text { 2017, } & 13 & \text { (7), }\end{array}$ https://doi.org/10.1371/journal.pcbi.1005659.

(48) Levine, B. G.; Coe, J. D.; Martínez, T. J. Optimizing Conical Intersections without Derivative Coupling Vectors: Application to Multistate Multireference Second-Order Perturbation Theory (MS-CASPT2) †. J. Phys. Chem. B 2008, 112 (2), 405-413. https://doi.org/10.1021/jp0761618.

(49) Levine, B. G.; Martínez, T. J. Isomerization through Conical Intersections. Annu. Rev. Phys. Chem. 2007, 58, 613-634. https://doi.org/10.1146/annurev.physchem.57.032905.104612.

(50) Levine, B. G.; Martínez, T. J.; Mart1, T. J. Ab Initio Multiple Spawning Dynamics of Excited Butadiene: Role of Charge Transfer. J. Phys. Chem. A 2009, 113 (46), 12815-12824. https://doi.org/10.1021/jp907111u.

(51) Saltiel, J.; Krishna, T. R. S.; Laohhasurayotin, K.; Ren, Y.; Phipps, K.; Davis, P. H.; Yee, W. A. Medium Effects on the Direct Cis-Trans Photoisomerization of 1,4-Diphenyl-1,3-Butadiene in Solution. J. Phys. Chem. A 2011, 115 (11), 2120-2129. https://doi.org/10.1021/jp111482m.

(52) The Gas-Phase MECI Are below the S1 Minimum like the MECI for Butadiene, but Unlike the MECI for Phenylbutadiene Which Are above the S1 Minimum.

(53) Wang, B.; Wang, X.; Wang, W.; Liu, F. Exploring the Mechanism of Fluorescence Quenching and Aggregation-Induced Emission of a Phenylethylene Derivative by QM (CASSCF and TDDFT) and ONIOM (QM:MM) Calculations. J. Phys. Chem. C 2016, 120 (38), 21850-21857. https://doi.org/10.1021/acs.jpcc.6b07963.

(54) Peng, X. L.; Ruiz-Barragan, S.; Li, Z. S.; Li, Q. S.; Blancafort, L. Restricted Access to a Conical Intersection to Explain Aggregation Induced Emission in Dimethyl Tetraphenylsilole. J. Mater. Chem. C 2016, 4 (14), 2802-2810. https:/ / doi.org/10.1039/c5tc03322e.

(55) Sumita, M.; Saito, K. Theoretical Study on Hula-Twist Motion of Penta-2,4-Dieniminium on the S1 Surface under Isolated Condition by the Complete Active Space Self-Consistent Field Theory. Chem. Phys. Lett. 2006, $424 \quad$ (4-6), 374-378. https://doi.org/10.1016/j.cplett.2006.04.093.

(56) Boggio-Pasqua, M.; Ravaglia, M.; Bearpark, M. J.; Garavelli, M.; Robb, M. a. Can Diarylethene Photochromism Be Explained by a Reaction Path Alone? A CASSCF Study with Model MMVB Dynamics. J. Phys. Chem. A 2003, 107 (50), 11139-11152. https://doi.org/10.1021/jp036862e. 
1

2

3

4

5

6 TOC Graphic

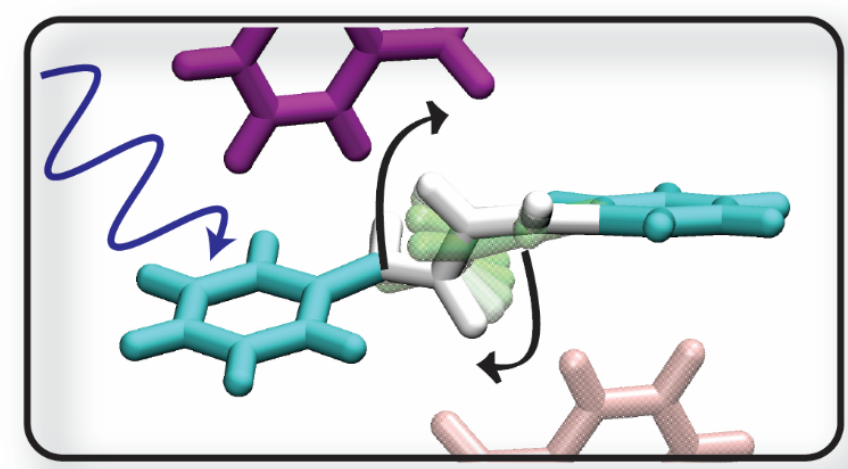

7 